\title{
OSTI
}

\section{SATURATION OF THE ION TRANSVERSE INSTABILITY*}

\author{
S. Heifets
}

Stanford Linear Accelerator Center, Stanford University, Stanford, CA 94309

\begin{abstract}
Fast Ion Instability is studied in the nonlinear regime. It is shown that exponential growth of the linear regime is replaced in this case by the linear dependence on time. Numeric and analytical results are presented describing the beam profile and the beam spectrum in both regimes.
\end{abstract}

\section{MASTER}

DISTRIBUTION OF THIS DOCUMENT IS UNLIMITED

The full version of the paper contributed to PAC 1997, Vancouver, Canada May 2 - May 6, 1997

\footnotetext{
${ }^{*}$ Work supported by Department of Energy contract DE-AC03-76SF00515.
} 


\section{DISCLAIMER}

This report was prepared as an account of work sponsored by an agency of the United States Government. Neither the United States Government nor any agency thereof, nor any of their employees, make any warranty, express or implied, or assumes any legal liability or responsibility for the accuracy, completeness, or usefulness of any information, apparatus, product, or process disclosed, or represents that its use would not infringe privately owned rights. Reference herein to any specific commercial product, process, or service by trade name, trademark, manufacturer, or otherwise does not necessarily constitute or imply its endorsement, recommendation, or favoring by the United States Government or any agency thereof. The views and opinions of authors expressed herein do not necessarily state or reflect those of the United States Government or any agency thereof. 


\section{DISCLAMMER}

Portions of this document may be illegible in electronic image products. Images are produced from the best available original document. 


\section{Introduction}

The Fast Ion Instability discovered recently [1] has been studied numerically and confirmed experimentally [2]. The transverse instability is caused by the interaction of a train of bunches with the residual gas. Ions produced by transversely offset bunches in the head of a train induce oscillations of the tail of the train. The ions may be cleared out by a gap after one revolution, but the memory remains in the train. Amplitude of oscillations initially grows exponentially as $\exp \sqrt{t / t_{c}}$ until the amplitude of a bunch centroid is of the order of the transverse rms $\sigma$ of a bunch. The initial rise time of the oscillations of a bunch centroid was found to be a fraction of a millisecond, even taking into account the spread of ion frequencies [3]. This is too fast to be observed in experiments directly.

The exponential regime is limited by the nonlinearity of the beam-ion interaction. As a result, exponential growth at large amplitudes is replaced by a linear dependence of the amplitude on time [4], and only this nonlinear regime can be observed experimentally.

The dynamics of the instability in the nonlinear regime is quite complicated. Additional to the nontrivial interference of the perturbations of the beam by the ions excited by different bunches in the train, the instability in the nonlinear regime essentially depends on the feedback damping and noise in the system while experiments without feedback are hardly possible due to the adverse effects of traditional multibunch instabilities. All that make necessary numerical studies of the instability.

Initial simulations of the instability were carried out with large number of macro particles in each bunch and realistic beam optics. This simulations show clearly exponential growth of the instability at small amplitudes and transition to the nonlinear regime at the amplitudes comparable with the transverse rms of a bunch. However, the simulations are quite time consuming and limited to short period of time. In this paper we present results of simulations based on much simpler model but extended to longer period of time. Simulations include also effects of the feedback and random noise describing the time dependence of the train profile and the beam spectrum.

\section{Analysis of Instability}

Consider a train of $n_{b}$ bunches equally spaced with the bunch spacing $s_{b}=c \tau_{b}$ in a ring with the circumference $C=2 \pi R=n_{t} s_{b}$. At the moment $t$ after $k$ revolutions, the $n$-th bunch is located at the position $s$ around the ring, $0<s<2 \pi R$, having transverse offset $y(t, z)$ where $z=n s_{b}$ is the distance of the bunch from the head of the train. Time $t$ and location $s$ are related: $t=k T_{r}+s+z$, where $T_{r}$ is the revolution time. A bunch passing location $s$ in the ring at the moment $c t_{0}=2 \pi R k+s+z$ may generate a group of ions centered at $Y\left(t_{0}, s, z\right)=y\left(t_{0}, z\right)$ and with negligible initial velocity. Motion of the ion centroid at $t>t_{0}$ is defined by the interaction of ions with the following bunches. As a result, ions are trapped, and centroid of ions $Y(t, s, z)$ oscillates in time during the passage of the train. Later ions are cleared by a gap in the train. Such a simple picture neglects decoherence of ions within the group. This effect has been discussed in [3].

Initial distribution of ions is defined by the density of the parent bunch which is Gaussian 
with $\mathrm{rms} \sigma_{x}, \sigma_{y}$, in the horizontal and vertical planes, respectively. We assume a flat beam $\sigma_{x} \gg \sigma_{y}$. Due to the shape of the bunch, the interaction is strongly suppressed at large distances between bunch and ion centroids. As the result, the vertical motion of electrons of the $n$-th bunch on the $k$-th turn is described by the equation [4]

$$
\frac{\partial^{2} y(t, z)}{\partial t^{2}}+\omega_{b}^{2} y(s, z)=-\kappa \sigma_{y} \int_{0}^{z} d z^{\prime} f\left[y(t, z)-Y\left(t, c t-z-k C, z^{\prime}\right)\right]
$$

where $\omega_{b}$ is betatron frequency,

$$
\kappa=\frac{4 r_{e}}{\gamma s_{b} \sigma_{x} \sigma_{y}} \frac{d N_{i}}{d s}
$$

and $d N_{i} / d s$ is the ion production rate per bunch proportional to pressure $p$ and the ionization cross-section $\sigma_{I}$. At $\sigma_{I}=2 \mathrm{Mbarn}$ and room temperature,

$$
\frac{d N_{i}}{d s}=0.06\left(\frac{p}{\text { torr }}\right) N_{b} \quad \mathrm{~cm}^{-1} .
$$

The function $f(\xi)$ in the RHS of Eq. (1) is either $f(\xi)=\xi / \sigma_{y}$ for $|\xi|<1$ (the linear regime), or $f(\xi)=\xi /|\xi|$ for $|\xi|>1$, in saturation regime, and depends only on the sign of $\xi$.

Similarly, the motion of the ions is described by the equation

$$
\frac{\partial^{2} Y(t, s, z)}{\partial t^{2}}=\omega_{i}^{2} \sigma_{y} f[y(t, c t-k C-s)-Y(t, s, z)]
$$

Here ion frequency

$$
\omega_{i}^{2}=\frac{2 N_{b} r_{p}}{A s_{b} \sigma_{y} \sigma_{x}} .
$$

depends on the atomic number of the residual gas $A$ and the rms of the beam $\sigma_{x, y}$ which may vary around the ring with the variation of the betatron function.

In the linear regime, these equations were considered in the original paper [1]. In this case, the solution is

$$
\begin{gathered}
y(t, z)=a(t, z) e^{i\left(\omega_{b} \pm \omega_{i}\right) z / c-i \omega_{b} t}+c . c ., \\
Y(t, s, z)=A(t, s, z) e^{-i \omega_{b} s / c \pm i \omega_{i}(t-s / c)}+c . c . .
\end{gathered}
$$

Note that for these choice of the phases, both terms in the RHS of Eq.(1) have equal phases, and the same is true for Eq. (4).

The equations for the amplitudes can be obtained by averaging over the fast oscillations

$$
\begin{gathered}
\frac{\partial a(t, z)}{\partial t}=\frac{i \kappa}{2 \omega_{b}} \int d z^{\prime} A\left(t, c t-z-k C, z^{\prime}\right), \\
\frac{\partial A(t, s, z)}{\partial t}= \pm \frac{\omega_{i}}{2 i} a(t, c t-k C-s) .
\end{gathered}
$$


For large $c t>>z$ these equations can be reduced to

$$
\frac{\partial a(t, z)}{\partial t}= \pm \frac{\kappa \omega_{i}}{4 \omega_{b}} \int_{0}^{z} z^{\prime} d z^{\prime} a\left(t, z^{\prime}\right) .
$$

The solution grows in time only for the upper sign

$$
a(t, z)=a_{0} e^{\sqrt{t / t_{c}}}, \quad \frac{1}{c t_{c}}=\frac{\kappa \omega_{i} z^{2}}{4 \omega_{b}},
$$

with the quasi-exponentially growth found in the original paper [1]. This solution corresponds to the wave $y_{b}(t, z) \propto \cos \left(\left(\omega_{b}+\omega_{i}\right) z / c-\omega_{b} t\right)$, see Eq. (7), while another solution $y_{b}(t, z) \propto \cos \left(\left(\omega_{b}-\omega_{i}\right) z / c-\omega_{b} t\right)$ is damped in time. Correspondingly, the spectrum of the BPM signal

$$
V(t) \propto \sum_{n, k} \delta\left(t-k C-n s_{b} / c\right) y\left(t, n s_{b}\right)
$$

consists of the betatron side-bands at frequencies $\omega=l \omega_{r} \mp \omega_{b}, l=0,1$.. with the envelope proportional to

$$
\left|\frac{\sin \left(\omega \mp \omega_{i}\right) \tau_{b} n_{b} / c}{\left(\omega \mp \omega_{i}\right) \tau_{b}}\right| .
$$

The envelope is centered at the ion frequency with the lower side-bands having amplitudes larger than that of the upper side-bands and growing in time. ${ }^{\dagger}$

In the nonlinear regime, the RHS in the Eqs. (1) and (4) depends on the function $f(\xi)$. We can expect that the variation of the argument $\xi$ in time is similar to variation of the RHS in the linear regime, that is proportional to $e^{i\left(\omega_{b} \pm \omega_{i}\right) z / c-i \omega_{b} t}$ in the equation for $y(t, z)$ and $e^{-i \omega_{b} s / c \pm i \omega_{i}(t-s / c)}$ in the equation for $Y(t, s, z)$, see Eq. (7). In the linear regime, the main harmonics in the RHS of Eq. (1) and Eq. (4) have frequency equal to the betatron or ion frequencies, correspondingly. In the strongly nonlinear regime, the spectrum of the RHS is a spectrum of a step-function which changes sign with the periods of betatron or ion oscillations. The spectrum of $f(\xi)$ in Eq. (1) and Eq. (4)) contains in this case harmonics of $\omega_{b}$ and $\omega_{i}$ correspondingly. The amplitudes of the harmonics roll off slowly as $1 / n$ for the $n$-th harmonic. In the nonlinear regime, the ions motion is a superposition of ion frequency harmonics. The amplitudes of harmonics don't grow in time but, without the feedback system, their number does. The RHS of Eq. (1) has always a harmonic oscillating with the betatron frequency. As the result, the amplitude of the bunch centroid motion linearly increases in time.

$$
A\left(n_{b}\right) \simeq \frac{\kappa s_{b}^{2} n_{b} n_{t} \beta_{y}}{2} \frac{t}{T_{r}} .
$$

The linear growth described by Eq. (14) replaces the quasi-exponential growth of the linear regime, see Eq. (11), when amplitude is of the order of transverse beam size rms. We can expect that the spectrum in the nonlinear regime would have envelope centered not only at ion frequency but also at all frequencies $n \omega_{i}+m \omega_{b}$ with integers $n$ and $m$. The number of side envelopes increase in time and spectrum become flatter.

\footnotetext{
${ }^{\dagger *}$ This result was obtained before by G. Stupakov.
} 


\section{Model for simulations}

To simulate the instability we use a simplified model describing each bunch in a train of $n_{b}$ bunches as a single macroparticle which goes around the ring in steps equal to $s_{b}$. At each step in time, all bunches, first of all, update their coordinates by rotation by the angle $\omega_{b} \tau_{b}$ in the phase plane of dimensionless coordinate $y_{b}=y / \sigma_{y}, y_{b}^{\prime}=y^{\prime} / y_{0}^{\prime}$. Here $y_{0}^{\prime}=\sigma_{y} / \beta_{y}$ is natural divergence of the beam, and $\beta_{y}=c / \omega_{b}$. In the same way, the coordinates of the ion centroids $Y_{i}=Y / \sigma_{y}$ is changed to $Y_{i}+Y_{i}^{\prime}$, where $Y_{i}^{\prime}=(d Y / d t) \tau_{b}$, and $Y_{i}^{\prime}$ remains at this step unchanged. Then all bunches get a kick from each group of ions at the new location of individual bunches

$$
\bar{y}_{b}^{\prime}=y_{b}^{\prime}-\kappa s_{b}^{2} \beta_{y} f\left(y_{b}-Y_{i}\right)
$$

where $f(x)=x$ if $|x|<1$ or $f(x)=x /|x|$ if $|x|>1$.

Similarly, each group of ions gets a kick

$$
\bar{Y}_{i}^{\prime}=Y_{i}^{\prime}+\left(\omega_{i} \tau_{b}\right)^{2} f\left(y_{b}-Y_{i}\right)
$$

Then, each bunch generates an ion macroparticle with the offset equal to the offset of a bunch, all ions are killed at the location of the ring just left by the last bunch in the train, and the process repeats again. At each step, there are $n_{b}$ macroparticles representing bunch centroids and, at maximum, there is the same number of macroparticles in each of $n_{b}$ locations around the ring representing ions. To model variation of the rms beam size around the ring, the ion frequencies and the kicks to the bunches are periodically modulated with the period equal to 1/12-th of the circumference of the ring (periodicity of the ALS lattice). The feedback was modeled as a single additional kick for each bunch per turn with the amplitude proportional to the offset of the same bunch calculated at the location shifted upstream by the betatron phase $\pi / 2$

$$
\bar{y}_{b}^{\prime}(s)=y_{b}^{\prime}(s)+g y_{b}\left(s-\pi \beta_{y} / 2\right) .
$$

The gain $g$ defines amplitude damping time $\tau_{d}=2 T_{r} / g$. Random kick uniformly distributed within the range $\pm a_{n s}$ was added to the RHS of Eq. (17) to simulate noise.

The amplitudes of bunches in the train were plotted giving the snap-shot of the beam profile. Offsets of all bunches were stored at a given location and then FFT was used to produce the snap-shot of the beam spectrum.

Most of the simulations were carried out for the ALS-like ring with the revolution period $T_{r}=n_{t} \tau_{b}, n_{t}=328, \tau_{b}=2 \mathrm{~ns}$, for the bunch train of $n_{b}=50$ bunches, and He gas $(A=4)$. The bunch parameters were: $N_{b}=4 \times 10^{9}, \sigma_{x}=165 \mu$, and $\sigma_{y}=27 \mu$. The betatron tune was $\nu_{y}=8.18$, and the ion frequency with these parameters was $50.8 \mathrm{MHz}$. The pressure was increased to $2 \mu$ Torr and the damping time of the feedback system to $\tau_{d}=0.1 \mathrm{~ms}$ to speed up simulations. Initially there were no ions in the ring, and initial conditions were $y_{b}=y_{b}^{\prime}=0$ for all but the first bunch, for which initial offset of $y_{b}=1.0 \times 10^{-4}$ (in units of $\left.\sigma_{y}\right)$ and $y_{b}^{\prime}=0$ were taken. Results for different amplitude of the noise $a_{n s}$ and modulation $\Delta \omega_{i} / \omega_{i}$ are described below. 


\section{Results of the simulations}

Fig. 1 shows growth of the amplitude of the last bunch in the train with number of turns. Dependence is shown in logarithmic and natural scales. In the left hand side, results are shown with the feedback system turned down $\left(1 / \tau_{d}=0\right)$, without noise (amplitude of the noise $a_{n s}=0$ ) and with the amplitude of modulation of ion frequencies $\bmod =\Delta \omega_{i} / \omega_{i}=0$ or $\bmod =0.5$. Initially, result clearly corresponds to the quasi-exponential growth of the linear regime with the parameter $t_{c}=0.41 \mu s$ in accordance with Eq. (11. Later, the growth of the dimensionless amplitude is only linear with time and in agreement with Eq. (17), which gives the rate $d A / d n=0.05$.

Results with the feedback turned on $\left(\tau_{d}=0.1 \mathrm{~ms}\right)$ are shown in the right hand side of Fig. 1. After initial growth, the amplitude of the last bunch oscillates around some steady level. It is interesting that the steady level is lower although the initial growth is faster for the higher noise level. This is, probably, because the effect of the noise is two-fold: noise excites bunch oscillations but at the same time decohere oscillations of different bunches preventing fast growth of the collective ion instability. Effect of the ion frequency modulation in the saturation is small, see two curves without modulation and with the amplitude of modulation $\bmod =0.5$.

Results on the beam profile are shown in Fig. 2 and Fig. 3. Initially, the amplitude of a bunch grows according to the linear theory and much faster for the bunches in the tail of the train then in the head. At a given moment, we look for a bunch which is the first in the train (starting from the head) to have a given amplitude, $A=1$. In the beginning, for small initial amplitude $y(0)$ taken for simulations, there are no such bunches at all. After several hundred turns such a bunch can be found in the tail of the train. The bunch number with $A=1$ then decreases because bunches closer to the head of the train reach the given level of excitation. Later, however, the feedback takes over and suppresses oscillations of the bunches in the head of the train to zero amplitudes. As a result, the growth rate and the amplitudes of the following bunches decrease and the bunch number with the amplitude $A=1$ increases in time. Oscillations with large amplitudes retain only in the very tail of the train and, eventually, all oscillations are damped out.

If we now, additionally to the feedback, turn on the noise, the beam profile goes to a steady-state, see Fig. 3. Without the instability, the equilibrium amplitude of a bunch in units of $\sigma$ would be

$$
A_{\infty}=\sqrt{y_{b}^{2}+y_{b}^{\prime 2}}=\sqrt{a_{n s}^{2} \tau_{d} / 6 T_{r}} .
$$

For the parameters used in simulations, $T_{r}=0.656 \mu \mathrm{s}$ and $\tau_{d}=0.1 \mathrm{~ms}$, this amplitude corresponds to the nonlinear regime $A_{\infty}>1$ for the amplitude of the noise $a_{n s}>0.2$ If the amplitude is smaller than that, the head of the train oscillates in the linear regime, and the transition to the nonlinear regime takes place somewhere closer to the train tail.

With the instability, the beam profile oscillates around almost triangular shape with amplitudes larger in the tail of the train. This beam profile was observed experimentally [5]. The steady-state amplitudes depend on the feedback and are smaller for smaller $\tau_{d}$. Comparison of the beam profile with different level of the noise shows that the maximum excitation of the beam is not monotonic function of the amplitude of the noise $a_{n s}$ and may be larger for 
smaller noise although it goes down again at larger $a_{n s}$. Possible explanation is mentioned above.

The ion frequency modulation reduces the rate of the instability [3]. Effect is quite noticeable in the linear regime, but affects less the steady state amplitudes which are mostly given by the relation between the feedback and the noise.

The beam spectrum at small number of turns has all features of the linear regime: envelope is centered at the ion frequency, $f_{i} / f_{r}=33.3$, see Fig. 4, and the upper side-bands have lower amplitudes then lower side-bands. On the longer time scale, the spectrum changes: more harmonics with frequencies $f_{i} \pm n f_{b}$ appear and the ion frequency decreases due to the increase of the amplitudes of ion. In the extreme nonlinear case, ions oscillate in a potential well $U=k_{i}^{2}|Y|$ and have frequencies depending on the amplitudes $A=\max \left(y_{i}\right)$,

$$
\frac{f}{f_{r}}=\frac{n_{t} \omega_{i} \tau_{b}}{4 \sqrt{2 A}}
$$

where $\omega_{i}$ is ion frequency in the linear regime. In the nonlinear regime, where the interaction between ions and bunches depend mostly on the sign of the relative position of the bunch and ion centroid, there is no reason to expect that the spectrum is centered around the ion frequency which is typical for the dipole signal of the linear regime. It should be noted, that for relatively low noise level, the head of the train can have small amplitudes corresponding to the linear regime while the tail of the train at the same time may be in the nonlinear regime.

The beam spectrum in the nonlinear regime with feedback and noise initially is much wider than that in the linear regime, Fig. 5, but with time only relatively few harmonics with low frequencies survive.

Calculations with the train of 100 bunches lead to similar results scaled correspondingly with the number of bunches.

\section{Conclusion}

Ion-induced fast transverse instability is constrained by nonlinear effects. Nonlinear effects stop quasi-exponential growth of the amplitude and only the linear with time growth remains. The feedback damping suppresses the bunch oscillations first in the head of the train, effectively reducing the train length and, therefore, the growth rate of the instability. With the noise, the beam takes the typical triangular shape with the profile determined by relation between noise and the feedback. The spectrum of the beam become wider and flatter comparing to the spectrum predicted by the linear theory. Details of the spectrum again depend on the noise and feedback. This may explain unstable character of the spectrum in the experiments.

\section{Acknowledgments}

I thank J. Byrd, A. Chao, G. Stupakov, F. Zimmermann for useful discussions. 


\section{References}

[1] T. Raubenheimer, F. Zimmermann. Interaction of a Charged Particle Beam with Residual Gas Ions or Electrons, SLAC, January, 1995

[2] J. Byrd, A. Chao, S. Heifets, M. Minty, T. Raubenheimer, J. Seeman, G. Stupakov, J. Thomson, F. Zimmermann, Measurements of a Fast BeamIon Instability at the ALS, PAC 1997, Vancouver, Canada

[3] G. Stupakov, T. Raubenheimer, F. Zimmermann. Effect of Ion Decoherence on Fast Beam-Ion Instability, SLAC, January, 1995

[4] S. Heifets, Saturation of the Ion Induced Transverse Blow-up Instability, SLAC-PUB6959, January 1996

[5] J. Byrd, Preliminary measurements of the FII, PAC 1996 

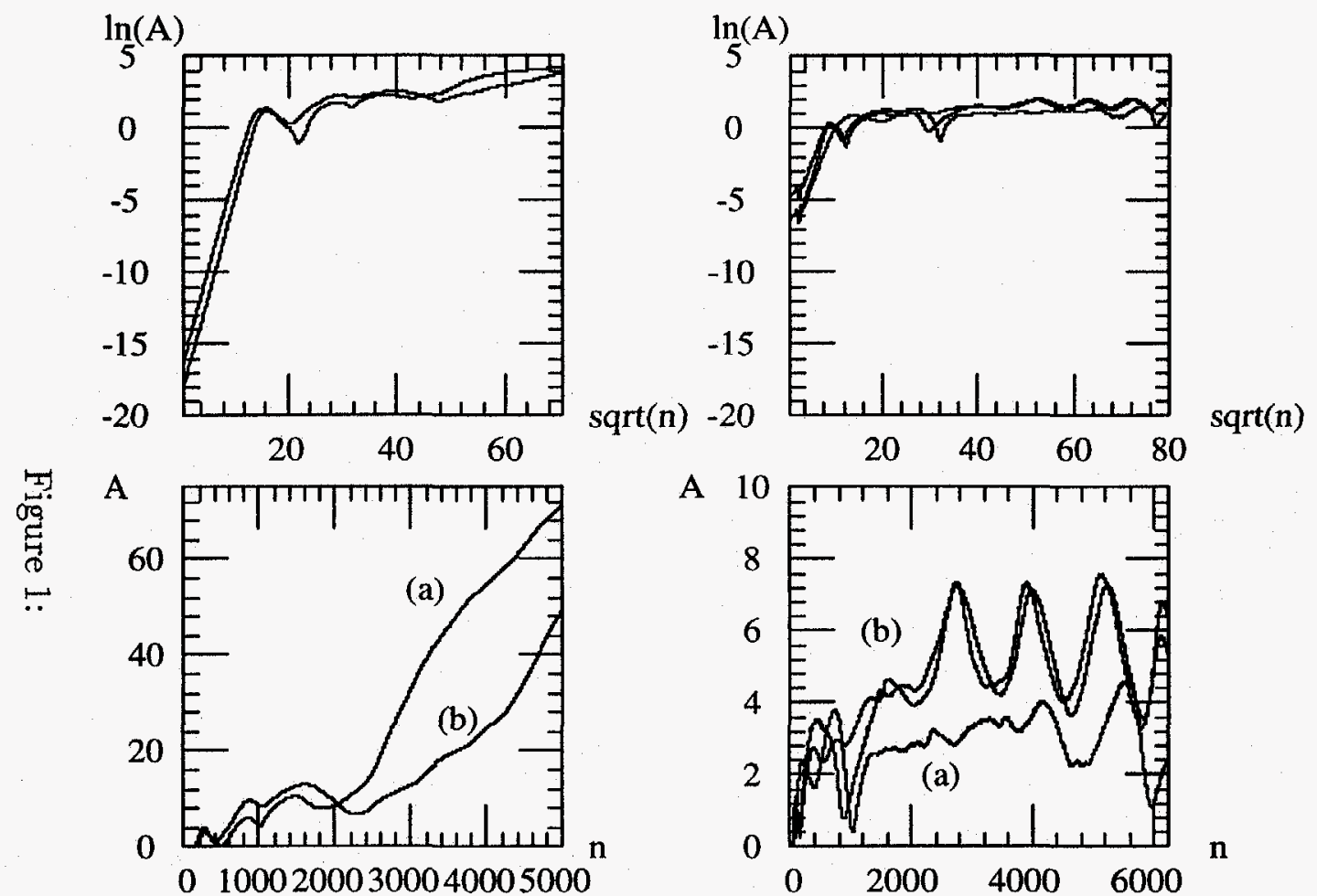

Amplit. of the last bunch vs number of turns in regular and log scales.

Note transition from exp. to linear growth.

(a) tau_fdb $=0.1 \mathrm{~ms}, \mathrm{a} \_\mathrm{ns}=0.01, \bmod =0.5$

Noise and feedback turned off

(b) tau_fdb $=0.1 \mathrm{~ms}$, a_ns $=0.002, \bmod =0.5$

(a) $\bmod =0$, (b) $\bmod =0.5$.

(dots) tau_fdb=0.1 ms, a_ns $=0.002, \bmod =0.0$ 


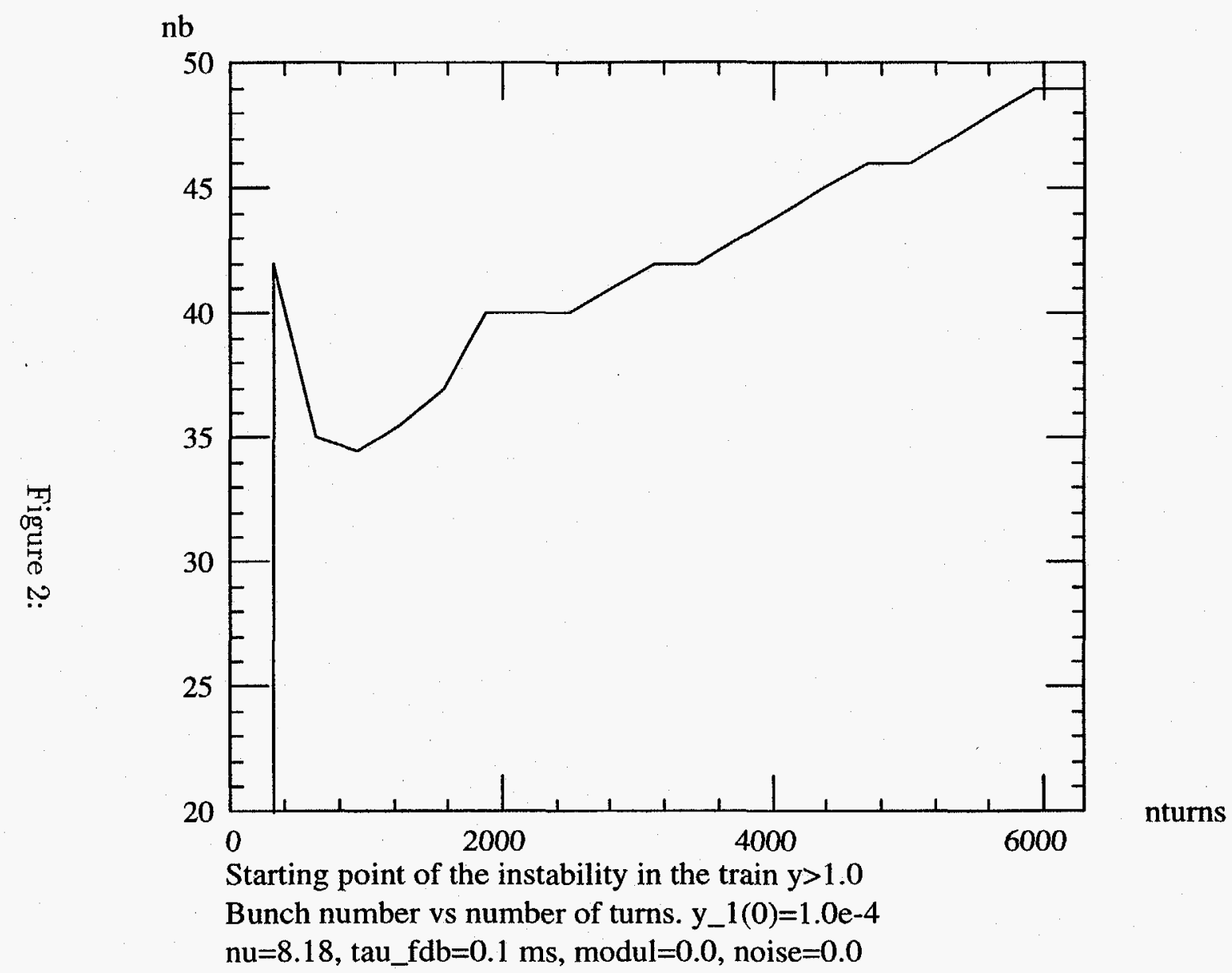



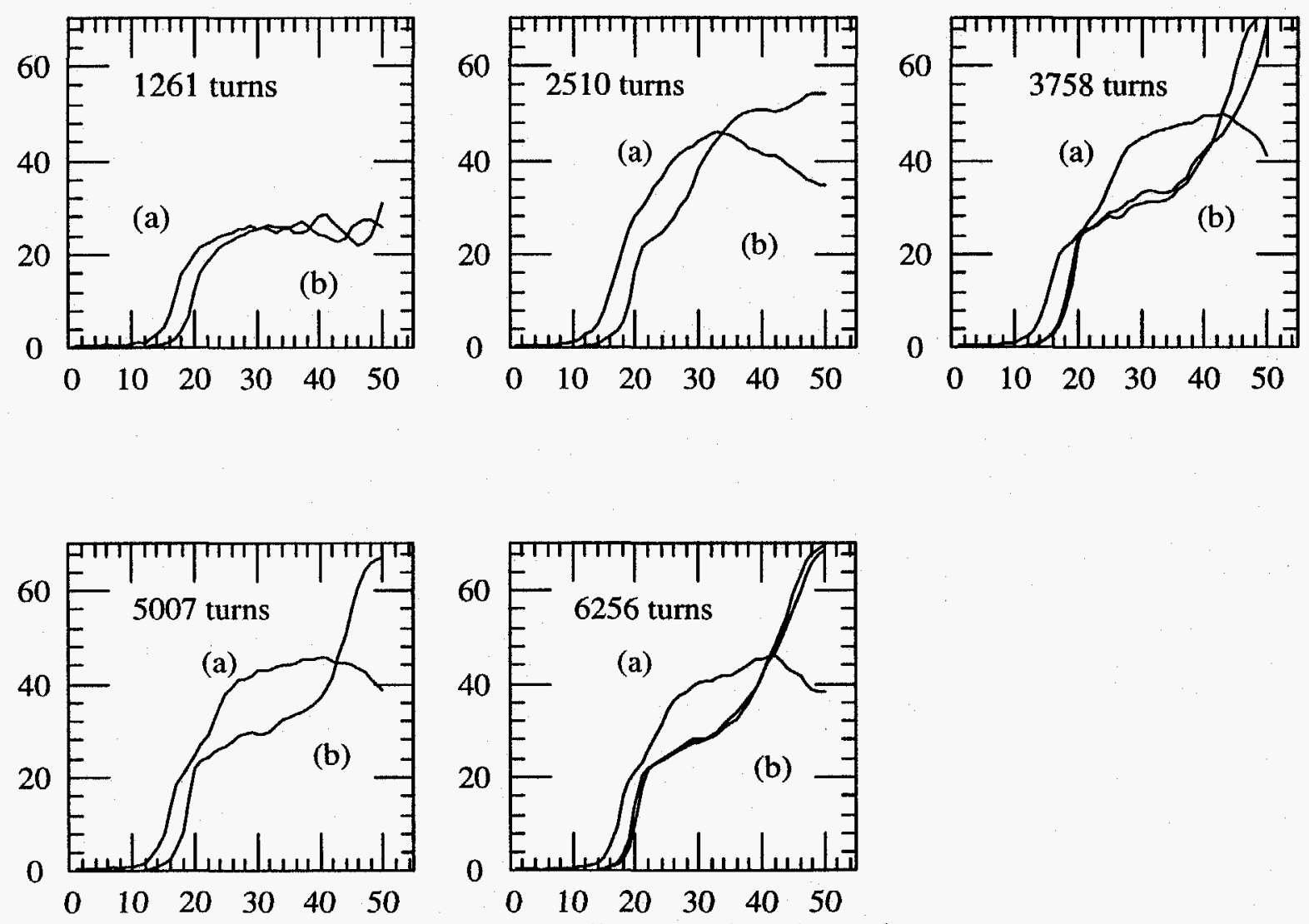

Snap-shot of the beam profile. Amplitudes vs bunch number Vertical scale is blown up in 12.5 times

tau_fdb $=0.1 \mathrm{~ms}, \mathrm{mod}=0.5$ (a) a_ns $=0.01$, (b) a_ns $=0.002$.

Curve a_ns $=0.002$, mod $=0.0$ shown in two cases

is about the same as in the case (b). 

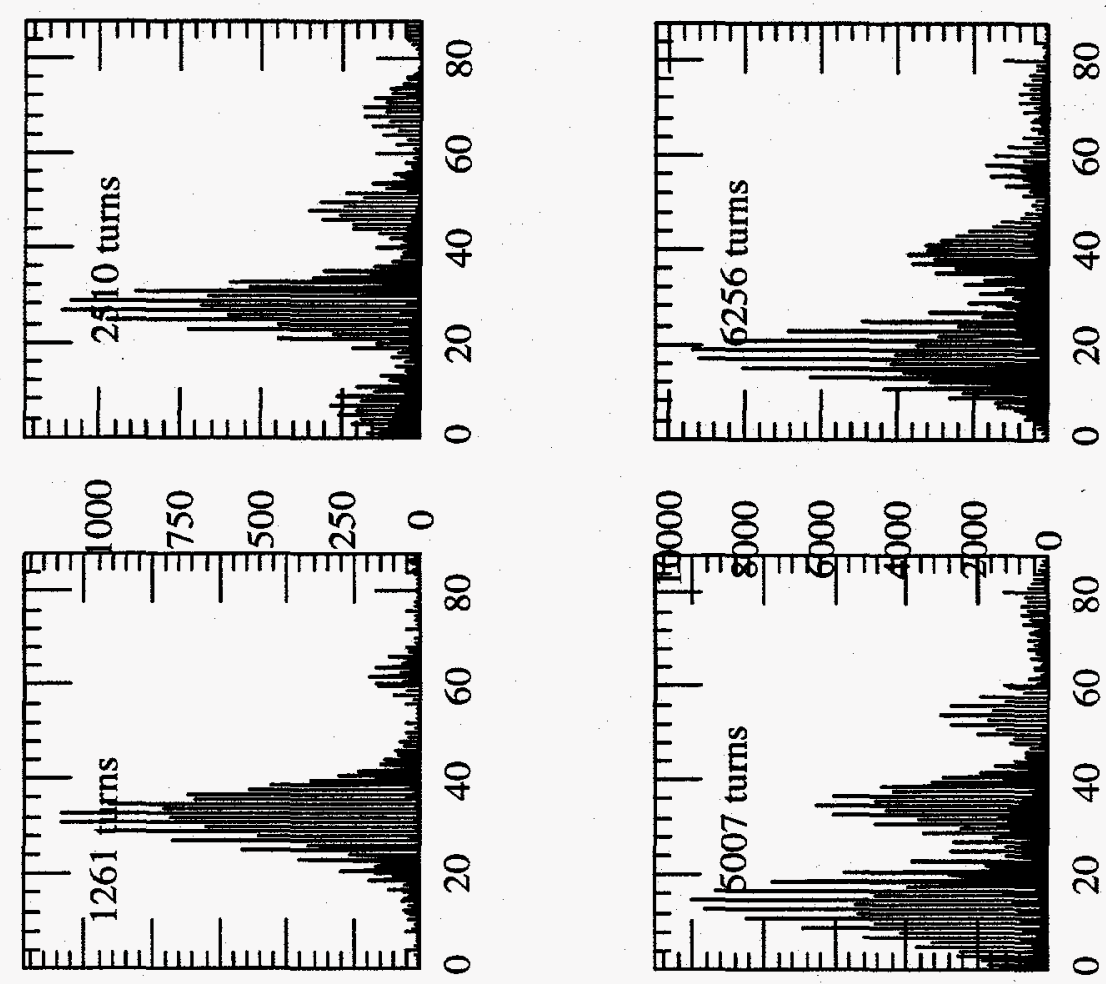

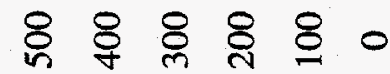
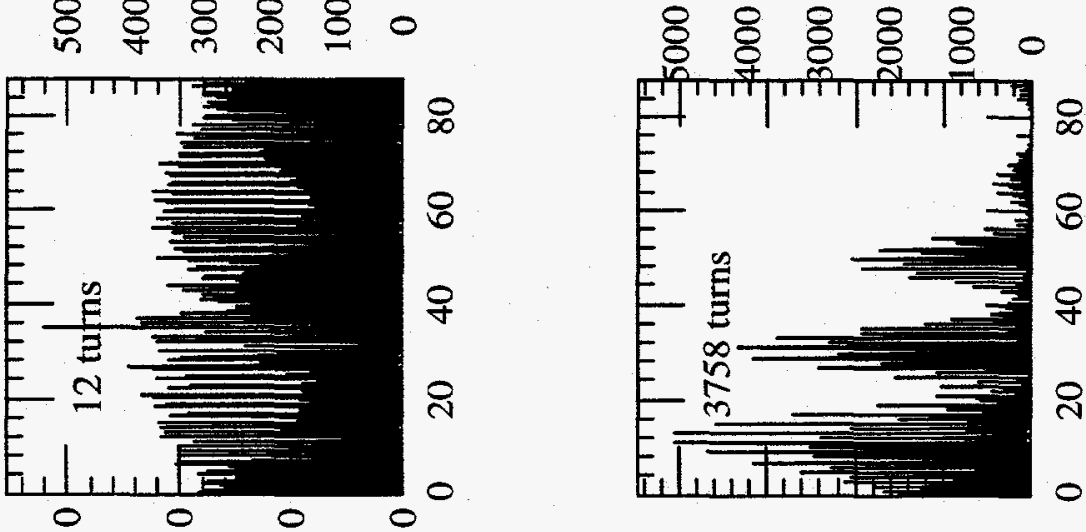

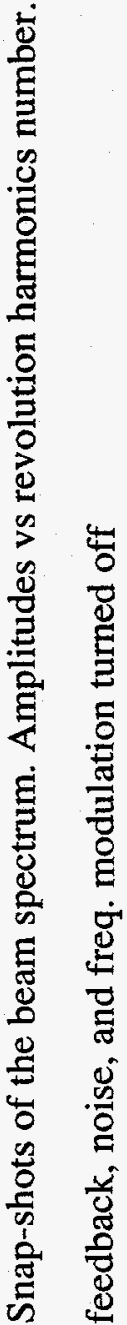

\&

Figure 4: 


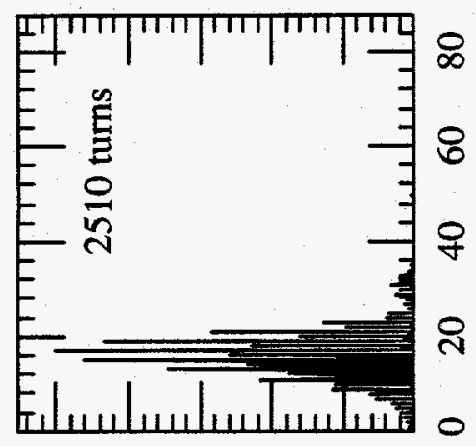

으용 응 응
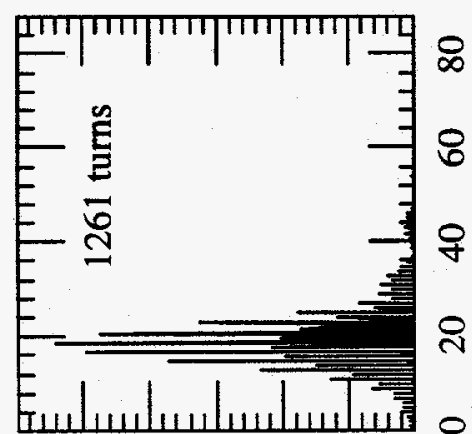

응응

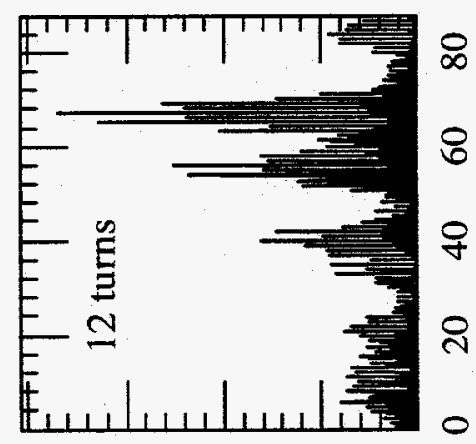

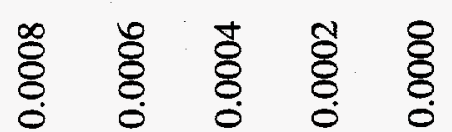
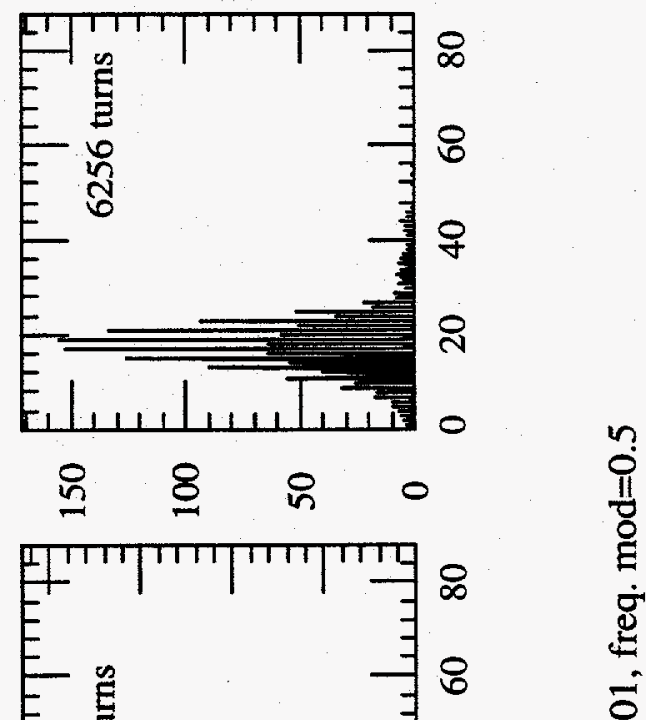

$E$

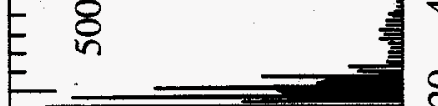

كسبسلس
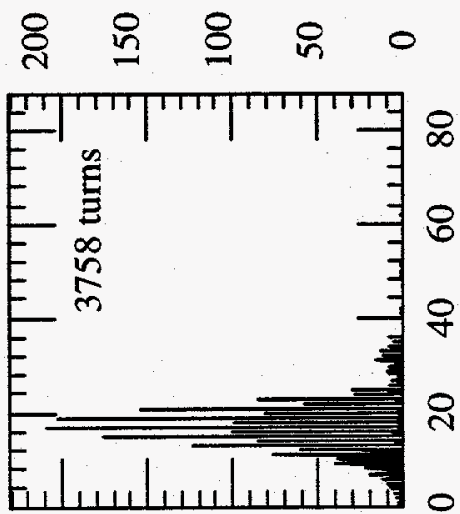

\& 8 응

Figure 5: 